\title{
An Examination of the Validity of Direct Product Perceptions
}

\author{
Michael D. Johnson \\ University of Michigan \\ David A. Horne \\ California State University at Long Beach
}

\begin{abstract}
The validity of a class of direct product perceptions is examined. Consumers recorded their perception of different products directly within existing spatial or treelike representations of the products' competitors. A conceptual model is developed that describes consumers' ability to provide direct perceptions within both space and tree representations for both brands and product categories. An empirical study is reported which supports the model and the validity of the recorded perceptions.
\end{abstract}

Similarity perceptions are central to a number of consumer research areas, from product categorization (Sujan, 1985) to consumer knowledge (Alba \& Hutchinson, 1987) to choice (Johnson, 1984). Traditionally, examinations of interproduct similarity have involved the spatial or tree scaling of pairwise proximity comparisons (e.g., Arabie, Carroll, DeSarbo, \& Wind, 1981; Cooper, 1983; Green, 1975; Srivaetava, Leone, \& Shocker, 1981). Yet the number of required comparisons may make similarity scaling cumbersome or restrictive in some research settings (Hauser \& Koppelman, 1979; Johnson, Lehmann, \& Horne, 1990). One alternative is to collect direct perceptions of interproduct similarity. The present study explores a particular class of direct perceptions which represents a hybrid of traditional similarity scaling and "direct" mapping. Accordingly, product space or tree representations may themselves be used as data collection devices; consumers may 
record their perception of a product's position directly within a similarity scaling representation.

our goal is to examine the validity and illustrate the scope of these direct perceptions. We use space and tree representations to collect direct perceptions for both categoryand brand-level stimuli. The procedure requires consumers to consider the overall similarity of some target product to other products in a representation and then translate this perception into a distance. We develop and test a conceptual model of this perceptual measurement process in order to assess the nomological validity of the resulting perceptions (Bagozzi, 1980; Cronbach \& Meehl, 1955).

\section{DIRECT PRODUCT PERCEPTIONS}

Research in psychology suggests a basic compatibility between scaled and direct perceptions (Baird, Merrill, \& Tannenbaum, 1979; Merrill \& Baird, 1979). Primarily these studies use Lynch's (1960) cognitive mapping technique where subjects directly sketch maps of an environment. For example, Baird, Merrill, and Tannenbaum (1979) compared multidimensional scaling (MDS) representations of subjects' pairwise distance judgments with their direct maps of building locations in an actual environment (a college campus). Both the pairwise judgments and the direct maps provided similar, accurate representations of the actual building locations. Merrill and Baird (1979) made a similar comparison involving the locations of facilities in an ideal town. Once again, both the MDS maps derived from pairwise judgments and the direct maps seemed appropriate to study the subjects' cognitive representations.

In many research settings the focus is on a particular purchase or consumption alternative under a number of different experimental conditions or at different points in time. In such cases direct perceptions may be combined with similarity scaling to facilitate the perceptual measurement process. Specifically, the geometric representations and tree structures of existing similarity scaling techniques may be used, not as ends in themselves, but as data collection devices.

A general procedure for collecting these direct perceptions can be described as follows:

(1) Use a traditional similarity scaling technique to obtain a suitable external representation (i.e., scaling solution) of the product or service alternatives in question.

(2) Remove the target product (if included) from the external representation. 
(3) Present the test consumers with the marketing stimulus of interest, such as a new product concept, a possible product name, promotional material for an existing target product, etc.

(4) Have the test consumers indicate their perception or" the target product's position directly within the external representation using an intuitive description of that representation.

Spaces may be described as pictures or maps and tree structures may be described intuitively as trees with branches. Subjects are asked to indicate a product's place in a map or branch location on a tree. (See Johnson \& Horne, 1990, for a more detailed discussion of the methodology.)

Johnson and Horne (1988) recently used spatial

representations in this manner to collect direct perceptions of products and services in a comparative advertising study. Similarly, Rosch (1975a) successfully used direct stimulus placements of geometric forms in a spatial representation to study cognitive reference points. Yet the validity of these direct perceptions remains unstudied. Moreover, previous studies have been limited to stimulus placements within spatial representations. It is unclear whether the technique can be applied using treelike representations of similarity. Both questions are addressed in the empirical study described below.

Direct perceptions are by no means all encompassing and were developed with particular data collection problems in mind. They are not a direct substitute for traditional similarity measures. Instead, they allow researchers to assess the overall perception of a particular product in those settings where sole reliance on paired comparison judgments is impractical. Perhaps the most obvious limitation of the procedure outlined here is that it can only be as good as the external representations used to collect perceptions. If a representation is not an accurate depiction of the test consumers' perceptions, any subsequent direct perceptions will be problematic.

\section{PERCEPTUAL MEASUREMENT MODEL}

The direct perceptions described herein require consumers to interact directly with a scaling solution. In this section of the article we develop a conceptual model of this interaction. Testing the model provides an examination of the validity as well as the scope of direct perceptual measurement. Specifically we explore the nomological validity of direct perceptions. Nomological validity, a form of construct validity, is the 
degree to which a theoretical variable or construct behaves as it should behave within a system of related constructs or nomological net (Ba-gozzi, 1980). That is, to scientifically clarify what something is, one must specify "the laws in which it occurs" (Cronbach \& Meehl, 1955, p. 290).

This approach to validity assessment is based on several considerations. It allows us to assess the validity of direct perceptions in their own right and not relative to other similarity measures. This is consistent with our view that direct perceptions are not meant to be a substitute for traditional similarity judgments; they are meant for those situations where complete reliance on paired comparison judgments is prohibitive. A more practical advantage is that the approach allows us to examine the relative usefulness of qualitatively different scaling representations for collecting perceptions at both the brand and category levels. Finally, as mentioned earlier, previous research already suggests a consistency between direct and scaled perceptions.

The theoretical construct at the heart of our conceptual model we call perceptual measurement. As a measure of perceived similarity, direct perceptions require consumers to consider how similar the target product is to the other products in an external representation and then translate this perceived similarity directly into a distance. This translation of a similarity perception into a distance is the key to a valid direct perception. Perceptual measurement is defined, therefore, as the degree to which a similarity-based product perception is translated into a distance in the external representation.

A second important and related construct is the difficulty of the task. A number of factors may reflect task difficulty, including the subject's ability to think in terms of the representation, whether the subject agrees with the distances among products in the representation, the subject's confusion with the task, and ease or enjoyment with which the subject performs the task.

Our conceptual model describes the effects that qualitatively different external representations and individual differences should have on these two important constructs. The model, presented in Figure 1, explains the perceptual measurement and the task difficulty of a direct perception as a function of two important distinguishing aspects of the external 
representation and three important individual differences, all of which are described in the following sections.

Notice that task difficulty has a direct, negative effect on perceptual measurement. The remaining constructs affect perceptual measurement both directly and indirectly through task difficulty.

\section{External Representation}

Consider first the inherent differences between trees and spaces. The treelike representations of hierarchical clustering (Johnson, 1967) and additive tree scaling (Sattath \& Tversky, 1977) provide a ' iable alternative (or complement) to perceptual spaces. However, one of the major advantages of spatial representations is their intuitive appeal and accessibility to the human eye (Shepard, 1972). From a naive subjects" standpoint, Euclidean spatial distance "as the crow flies" is an intuitive concept that may be easily related to their perception of similarity. Because we live in a dimensional world, spatial representations complement our perceptions of distance (Shepard, 1981). Thinking of product similarities as distances within or along the paths of a tree may not be so natural or complementary. Therefore, using spaces should increase perceptual measurement and decrease task difficulty relative to using trees (see Figure 1 ).

Using brand- or category-level stimuli to collect perceptions should also systematically affect perceptual

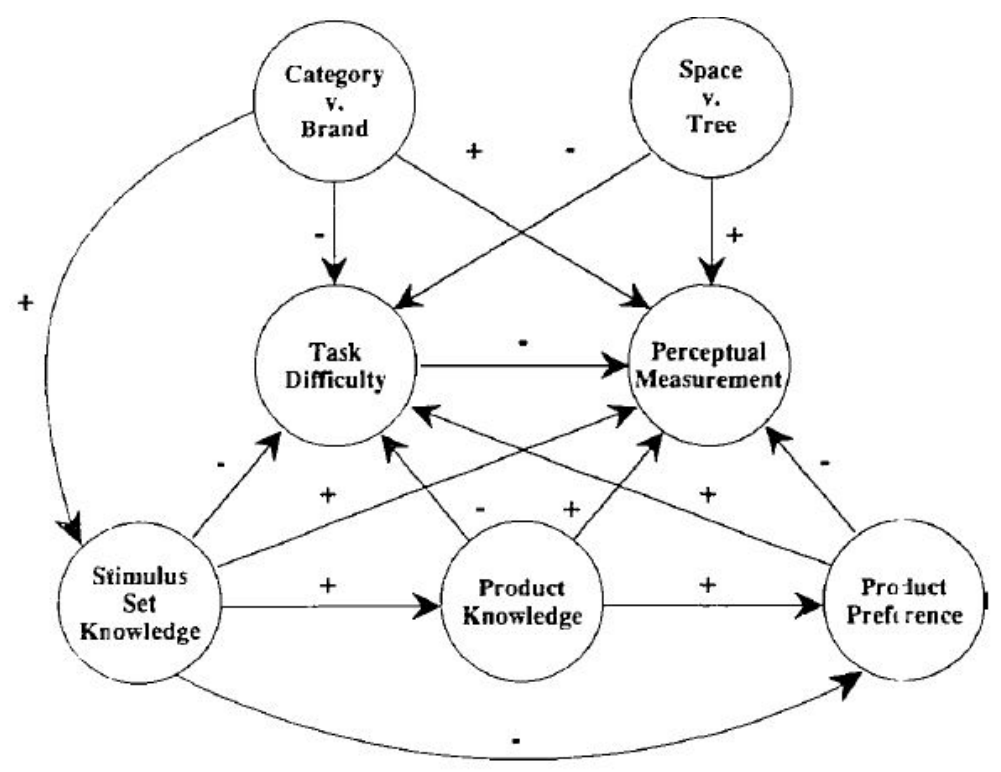

Figure 1. Perceptual Measurement Model measurement. Compared to brands, product categories such as soft drinks or candy bars are closer to the basic-level categories studied in psychology (Johnson \& Fornell, 1987). Product categories should, therefore, demonstrate higher degrees of inclusiveness or similarity of their members (Rosch, 1975a, 1975b; Rosch et al., 1976). This suggests that traditional 
produce categories are well defined and perceptually distinct relative to brands. Consider, for example, a subject who finds it rather straightforward just where to place popcorn relative to other snack foods in a space or tree, yet is unsure just where to place snickers relative to other candy bars. We thus predict that using perceptually distinct category-level stimuli will increase perceptual measurement and decrease task difficulty relative to using brands (see Figure 1).

\section{Individual Level Knowledge and Product Preference}

The model also incorporates the effects of individual differences in knowledge and preference on perceptual measurement and task difficulty. Regarding knowledge, we distinguish between the consumer's knowledge of the target product (product knowledge) and their knowledge of the entire set of products involved in the task (stimulus set knowledge). Product knowledge is necessary to understand and judge the object of comparison, whereas stimulus set knowledge provides an understanding of the reference group or context of the perception. We thus predict that both product knowledge and stimulus set knowledge should increase perceptual measurement as well as decrease task difficulty (see Figure 1).

Preference for the target product may also affect task difficulty and perceptual measurement. Product preference has the potential to bring nonperceptual factors into play. For example, the uniqueness and positive affect associated with a preferred product may make a direct perception of similarity difficult. Therefore, we predict that product preference decreases perceptual mesurement and increases task difficulty ( see Figure 1).

For completeness, the model incorporates the natural relationships involving the individual difference constructs. Following Howard (1977), category-level knowledge develops prior to brand-level knowledge. Thus our product category knowledge should, in general, exceed our knowledge of brands (see also Sujan, 1985). The results should be a direct positive relationship from the category versus brand nature of the stimuli to stimulus set knowledge. Stimulus set knowledge should, in turn, positively affect product knowledge. The greater our knowledge of a set of product stimuli, the greater should be our knowledge of any one member of that set. Finally, both product knowledge and stimulus set knowledge should affect product preference. The more knowledge we accumulate regarding the target product, the more likely we are to prefer and purchase that product. * Conversely, the greater our knowledge of 
the other products in the stimulus set, the lower should be our relative preference for the target product. Thus we posit a negative relationship from stimulus set knowledge to target product preference.

Overall we predict positive effects for spaces versus trees, categories versus brands, stimulus set knowledge, and product knowledge on perceptual measurement. These positive effects are both direct and indirectly mediated by task difficulty. In contrast, product preference should have a general negative effect on perceptual measurement.

\section{EMPIRICAL STUDY}

The study was conducted in two phases. The external representations were constructed in phase one. These representations were used in phase two to collect direct perceptions and test the model. In phase two, each subject was asked to indicate their perception of a single product within three qualitatively different representations, a spatial representation derived from MDS, and two tree-based representations, one derived from hierarchical clustering and one derived from additive tree scaling. The subjects were asked to provide verbal protocols while they performed the tasks (Ericsson \& Simon, 1980), Information was also collected regarding the subjects' knowledge, preferences, and task difficulty.

\section{Phase One}

In phase one, a convenience sample was asked to provide proximity judgments for products from one of five possible stimulus sets. Two of these sets involved brands from the same product category: soft drinks and candy bars. Three stimulus sets involved products that cross traditional product categories: beverages, snacks, and lunch products. Each set contained 12 product alternatives requiring subjects to make 66 paired comparisons. A total of 24, 24, 24, 24, and 27 subjects $\{\mathrm{n}=123$ ) rated the soft drink, candy bar, beverage, snack food, and lunch product stimuli, respectively. Each pair of alternatives was rated on a scale from $\odot$ (very dissimiliar) to 10 (very similar). Half of the subjects in each group rated the 66 pairs in one random order and the other half rated the same 66 pairs in the reverse order.

The judgments were pooled for each stimulus set and scaled using three qualitatively different techniques: multidimensional scaling (MDS) in two dimensions using the Euclidean metric 
(Roskam \& Lingoes, 1970), hierarchical clustering (HCS) using an average linkage method (Johnson, 1967), and additive tree scaling via ADDTREE (Sattath \& Tversky, 1977). The 15 different representations across the five different stimulus sets all provided reasonably good fits to the input data (Kruskal's stress for the solutions ranged from 0.04 to 0.13 ).

\section{Phase Two}

In phase two the MDS, HCS, and ADDTREE representation $s$ were used to collect direct perceptions for 10 products overall, two different products from each stimulus set. These included Pepsi Cola and Diet Orange Crush from the soft drinks, Snickers and Reese's Peanut Butter Cups from the candy bars, fruit juice and ice cream soda from the beverages, popcorn and cheese from the snack foods, and pizza and carrot from the lunch products. The direct perception procedure outlined earlier was followed for each product/space and product/tree combination.

A total of 198 subjects was asked to provide direct perceptions (approximately 20 subjects per product). Each subject indicated their perception of the target product within each of the three possible external representations. Given the possibility of carryover effects, half of the subjects in each product group provided their spatial perception first, and the remaining half provided their two tree-based perceptions first. Within each of these subgroups, the order of the HCS and ADDTREE representations was also counterbalanced, resulting in four order conditions.

Phase two was conducted using adult subjects who were recruited in a suburban mall of a major metropolitan area and were paid for their participation. Using a pencil-and-paper format, subjects first rated their confidence in evaluating each of the 12 alternatives from the stimulus set on a scale from $\odot$ (not at all confident) to 10 (very confident). Subjects then rated their attribute knowledge of each product on an absolute, 21-point scale which allows for comparisons across product categories (adapted from Johnson, 1984). Each subject then rankordered their preference for the 12 alternatives.

Subjects were then shown to an interview room where an experimenter individually ran each subject through the direct perception tasks and collected their verbal protocols. At the end of the experiment, the subjects were asked to rate separately the MDS, HCS, and ADDTREE tasks on 7-point agreedisagree scales regarding the following statements:

(1) I found it easy to think in terms of the map (tree). 
(2) I generally agreed with the distances among products in the map (tree).

(3) I found the task confusing.

(4) Placing the product in the map (tree) was easy.

(5) Placing the product in the map (tree) was fun.

\section{Verbal Protocol Coding and Analysis}

of the 594 task protocols collected in the study, some were excluded from the analysis. Following standard verbal protocol procedures, the experimenters were instructed only to prompt people to think aloud and, when the subjects were quiet, to ask them what they were thinking. In some unforeseen cases, an experimenter unduly coached or directed a subject and this data was excluded from further analysis. Equipment problems also caused the loss of some protocols. The end result was a total of 448 usable protocols and 535 usable direct perceptions.

Two judges who were naive regarding the research hypotheses divided the protocols into separate meaningful statements. Two judges (one carryover and one new, naive judge) then independently coded these statements into six general categories: (a) attribute, brand, or category based similarity/dissimilarity statements (e.g., "it is similar to. . . " "it tastes like. . ."), (b) distance statements (e.g., "it goes between... .," "it should be close to.. (c) positive/negative statements regarding the products and their placement, (d) statements eliciting satisfaction or dissatisfaction with the external representation, (e) statements regarding product usage occasions, and ( $f$ ) miscellaneous statements and task impressions (e.g., recalling past experiences, statements of understanding, pondering, etc.). The judges agreed in classifying over $89 \%$ of the statements and the discrepancies were resolved by discussion. After coding the statements in each protocol, the judges independently counted the number of products mentioned. The judges' agreement here was over $95 \%$ and discrepancies were again resolved by discussion. out of a total of 2,170 protocol statements, there were $1.203(55.4 \%)$ similarity statements and 401 (18.4\%) distance statements. Of the remaining statements, only 96 (4.4\%) were positive/negative statements regarding products, $55(2.5 \%)$ revealed satisfaction/dissatisfaction with the external representations, $54(2.4 \%)$ were statements regarding product use occasions, and $361(16.6 \%)$ were in the miscellaneous category.

Model Estimation 
Task difficulty is treated as a latent construct, with each subjects' responses to the five task difficulty statements of each direct perception serving as reflective indicators $\left(D_{1}-D_{5}\right)$. Three protocol-based measures operationalize latent perceptual measurement: the number of similarity statements $\left(M_{1}\right)$, the number of distance statements $\left(M_{2}\right)$, and the number of products considered $\left(M_{3}\right)$. These measures follow directly from our earlier definition of the construct. Perceptual measurement requires a subject to consider the various products involved $\left(\mathrm{M}_{3}\right)$, in terms of their similarity $\left(M_{1}\right)$, and translate this perception directly into a distance $\left(M_{2}\right)$. Our definition further suggests that $\left(M_{1}\right.$ $M_{3}$ ) be treated as formative indicators of perceptual measurement. Just considering similarities, for example, is not sufficient. These similarities must be translated into a distance.

Preliminary analysis of variance models revealed significant task difficulty and perceptual measurement differences between the spaces and the two trees, but not between the trees themselves. The model thus includes a dichotomous space versus tree variable $\left(S_{1}\right)$. A similar two-level variable captures the category versus brand level of the stimuli $\left(C_{1}\right)$. Stimulus set knowledge, which captures each subject's knowledge of all the products involved in the task, is modeled reflectively by two indicators: the subject's average self-rated confidence in evaluating the products in the set, and the subject's average self-rated attribute knowledge for the products $\left(\mathrm{K}_{1}\right.$ and $\left.\mathrm{K}_{2}\right)$. Product knowledge, which captures the subject's knowledge of the target product, is also modeled reflectively by two indicators, the subject's self-rated confidence in evaluating the target product, and the subject's self-rated attribute knowledge for the target product $\left(K_{3}\right.$ and $\mathrm{K}_{4}$ ). Preference is modeled reflectively by the subject's reverse rank-order preference for the target product $\left(P_{1}\right)$.

Ultimately the model focuses on the perceptual measurement construct which itself is modeled via formative indicators. The suggested estimation procedure in this case is partial least squares, which was performed here (Fornell \& Bookstein, 1982; Wold, 1982). The construct indicators were scaled such that all of the loadings should be positive.

\section{Model Results}

The indicator loadings and structural coefficients are presented in Figure 2. All of the indicator loadings are relatively large and positive supporting a sizable amount of valid variance in the measures. The relatively large loading for distance statements compared to that for similarity statements and products considered for the perceptual measurement construct 
is not surprising. Similarity statements pervaded the protocols, whereas the subjects' ability to translate their perceptions into a distance is the key to providing a direct perception. The model explains $85 \%$ of the covariance among the latent variables, which supports the hypothesized causal structure. The measurement model explains $56 \%$ of the covariance among the measurement variables.

Most important from a nomological validity standpoint is whether or not perceptual measurement and task difficulty are systematically related to and affected by the other constructs as predicted. The results reveal that 14 of the 15 structural coefficients are as predicted. Task difficulty had a negative effect on perceptual measurement. The use of spatial representations decreased task difficulty and increased perceptual measurement. This is consistent with spatial representations being a more intuitive and natural way to directly collect perceptions. Using categories as opposed to brands also increased perceptual measurement and decreased task difficulty. This is consistent with basic-level product categories being perceptually distinct relative to brands.

The use of categorical stimuli increased stimulus set knowledge, and the hypothesized relationships among the knowledge and preference constructs were well supported. Set knowledge positively affected product knowledge, which, in turn, positively affected product preference. Set knowledge also had a direct negative effect on product preference.

Finally, the results reveal individual difference effects on task difficulty and perceptual measurement. Stimulus set knowledge had a negative effect on task difficulty and a very small positive effect on perceptual measurement. Product knowledge also had a negative effect on task difficulty and a positive effect on perceptual measurement. These findings are consistent with the notion that knowledge improves a consumer's ability to provide direct perceptions. The predicted positive relationship between product preference and task difficulty also materialized. It appears that preference 


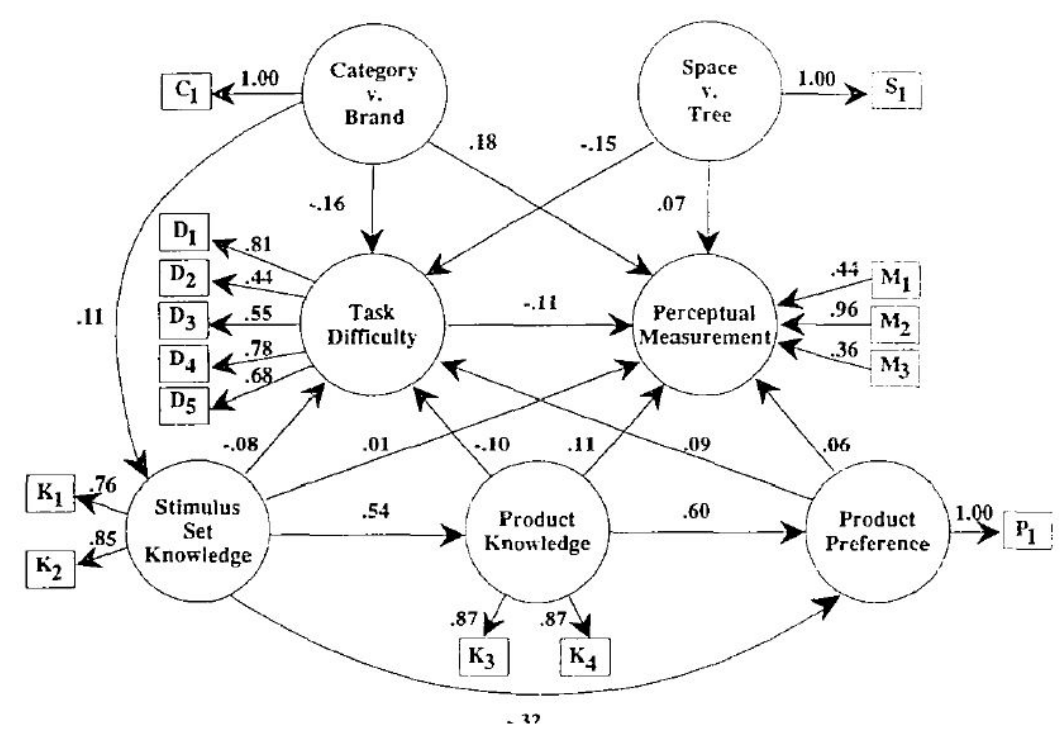

Figure 2. PLS Model Results

for the target product makes its direct perception more difficult. Not predicted was the observed positive effect for product preference on perceptual measurement. A possible explanation of this unexpected result is that preferred products sparked greater interest or motivation on the part of our subjects, increasing perceptual measurement.

The model results demonstrate the general positive effects of spatial representations, product category stimuli, and knowledge on perceptual measurement. These effects are both direct and mediated by task difficulty. The systematic nature of the results supports the conceptual model and the nomological validity of direct perceptions.

Yet the structural coefficients in Figure 2 are not particularly large. Consider, however, that the similarity and distance statements accounted for a majority of total protocol statements (74\%). Moreover, the average responses to the task difficulty questions, presented in Table 1, indicate that the subjects had no major difficulties with any of the external representations or stimuli.

These observations support the existence of ceiling effects for our task difficulty and perceptual measurement constructs which may explain the size of the coefficients. Thus even though systematic differences exist, the results suggest that both spaces and trees may be used to collect direct perceptions for brands as well as categories. 
Table 1. Differences in Task Difficulty.

\begin{tabular}{|c|c|c|c|c|c|}
\hline \multirow{2}{*}{$\begin{array}{l}\text { Dependent } \\
\text { Variable* }\end{array}$} & \multicolumn{3}{|c|}{ Representation } & \multicolumn{2}{|c|}{ Stimuli } \\
\hline & MDS & $\mathrm{HCS}$ & ADDTREE & Brands & Categories \\
\hline $\begin{array}{l}\text { Easy to think in } \\
\text { terms of } \\
\text { representation } \\
\text { Agreed with }\end{array}$ & 2.30 & 2.67 & 2.62 & 2.86 & 2.33 \\
\hline $\begin{array}{l}\text { Agreed with } \\
\text { distances in } \\
\text { representation } \\
\text { Found the task }\end{array}$ & 2.49 & 2.62 & 2.71 & 2.69 & 2.56 \\
\hline $\begin{array}{l}\text { Found the task } \\
\text { confusing } \\
\text { Placing the }\end{array}$ & 5.53 & 5.34 & 5.26 & 5.07 & 5.56 \\
\hline $\begin{array}{l}\text { product was } \\
\text { easy } \\
\text { Placing the } \\
\text { product was } \\
\text { fun }\end{array}$ & 2.12 & 2.61 & 2.43 & 2.65 & 2.23 \\
\hline
\end{tabular}

Rezponse scale range of 1 'agree to i ddisagreed.

\section{Direct Perceptions versus Initial Positions}

Finally, we compared the subjects' direct perceptions with the products' positions prior to their elimination from the representations. Using the products' initial, analytically derived positions as a benchmark is a heuristic way of assessing the face value of a direct perception. We measured the distance between each direct perception and the target product's scaled position in millimeters. (Distance in the trees was measured from where the subjects connected their branch for the target product to the connection of the target product's scaled branch.) These distances were compared with the two measures of stimulus set knowledge, the two measures of product knowledge, product preference, the three measures of perceptual measurement, and the five measures of task difficulty.

Given the interrelationships among many of these variables, separate linear models were estimated using each of the measures to explain the distance between the direct perceptions and the products' original positions. The results reveal significant negative correlations $(p<0.05)$ between the distance measures and each of the three perceptual measurement indicators, the number of similarity oriented statements $(r=-0.14)$, the number of distance-oriented statements $(r=-0.11)$, and the number of products overtly mentioned in the protocols $(r=-0.11)$. Put simply, the larger our perceptual measurement construct, the closer were the subjects' direct perceptions to 'he target products' scaled positions in the external representations. This result is consistent with the studies described earlier which 
demonstrate the complementarity of scaled maps and direct maps (Baird, Merrill, \& Tannenbaum, 1979; Merrill \& Baird, 1979). The only other significant relationship was an increase in distance with task confusion $(r=0.13, p<0.01)$.

\section{SUMMARY AND DISCUSSION}

The study and results provide two primary conclusions. First, direct perceptions of product alternatives do appear to tap consumer perceptions. Perceptual measurement varied systematically and predictably within our conceptual model supporting the validity of the perceptions. Second, while the differences between the spaces and the trees were systematic, they were not particularly large. It thus appears that both types of representations may be used to collect direct perceptions. The predominance of similarity and distance statements across the protocols and the low-rated difficulty of the space and tree tasks further support this conclusion.

The results provide insight into the application of direct perceptions. One of our more interesting findings is the potential advantage of using spatial representations in this type of task. Spatial representations are very intuitive and naturally lend themselves to collecting direct perceptions. There are similar potential advantages to using category-level stimuli. Product categories are perceptually distinct relative to brands. As a result, perceptual measurement increased and task difficulty decreased for the product categories. This suggests that direct perceptions may be particularly applicable to studies focusing on across-category competition.

Last, our results suggest that perceptual measurement increases with consumer knowledge. Both target product knowledge and stimulus set knowledge indirectly increased perceptual measurement by reducing task difficulty. Knowledge also directly increased perceptual measurement, particularly consumers' knowledge of the target product. As is the case with more traditional scaling methodologies, some minimum level of knowledge may be necessary to directly measure consumer perceptions. 


\section{REFERENCES}

Alba, J. W., \& Hutchinson, J. W. (1987). Dimensions of consumer expertise. Journal of Consumer Research, 13, 411-454.

Arabie, P., Carroll, J. D., DeSarbo, W. S., \& Wind, J. (1981). Overlapping clustering: A new method for product positioning. Journal of Marketing Research, 18. 310-317. Bagozzi, R. P. (1980). Causal models in marketing. New York: Wiley .

Baird, J. C., Merrill, A. A., \& Tannenbaum, J. (1979). Studies of the cognitive representation of spatial relations: II. A familiar environment. Journal of Experimental Psychology: General, 108, $92-98$.

Cooper, L. G. (1983). A review of multidimensional scaling in marketing research. Applied Psychological Measurement, 7, 427-450.

Cronbach, L. J., \& Meehl, P. E. (1955). Construct validity in psychological tests. Psychological Bulletin, 52, 281-302.

Ericsson, K. A., \& Simon, H. A. (1980). Verbal reports as data. Psychological Review, 87, 215-251.

Fornell, C., \& Bookstein, F. L. (1982). Two structural equation models: LISREL and PLS applied to consumer exit-voice theory. Journal of Marketing Research, 19, 440-452.

Green, P. E. (1975). Marketing applications of MDS: Assessment and outlook. Journal of Marketing, 39, 24-31.

Hauser, J. R., \& Koppelman, F. S. (1979). Alternative perceptual mapping techniques: Relative accuracy and usefulness. Journal of Marketing Re- search, 16, 495-506.

Howard, J. (1977). Consumer behavior: Application of theory. New York: McGraw-Hill.

Johnson, M. D. (1984). Consumer choice strategies for comparing noncomparable alternatives. Journal of Consumer Research, 11, 741-753.

Johnson, M. D., \& Fornell, C. (1987). The nature and methodological implications of the cognitive representation of products. Journal of Consumer Research, 14, 214-228.

Johnson, M. D., \& Horne, D. A. (1988). The contrast model of similarity and comparative advertising. Psychology \& Marketing, 5, 211-232.

Johnson, M. D., \& Horne, D.A. (1990). Direct perceptual measurement: Methodology and validity, Marketing Science Institute, Report No. 90-101, January.

Johnson, M. D., Lehmann, D. R., \& Horne, D. R. (1990). The effect of fatigue on judgments of interproduct similarity. International Journal of Research in Marketing, 7, 35-43.

Johnson, S.C. (1967). Hierarchical clustering schemes. Psychometrika, 32, 241-254. 
Lynch, K. (1960). The image of the city. Cambridge, MA: MIT Press.

Merrill, A. A., \& Biard, J. C. (1979). Studies of the cognitive representation of spatial relations: III. A hypothetical environment. Journal of Experimental Psychology: General, 108, 99-106.

Roskam, E., \& Lingoes, J. C. (1970). MINISSA-1: A FORTRAN IV (G) program for the smallest space analysis of square symmetric matrices. Behavioral Science, 15, 204-205.

Rosch, E. (1975a). Cognitive reference points. Cognitive Psychoogy, 7, 532- 547.

Rosch, E. (1975b). Cognitive representation of semantic categories. Journal of Experimental Psychology: General, 104, 192-233.

Rosch, E., Mervis, C. B., Gray, W. D., Johnson, D. M., \& BoyesBraem, P. (1976). Basic objects in natural categories. Cognitive Psychology, 8, 382-439. Sattath, S., \& Tversky, A. (1977). Additive similarity trees. Piychometrika, 42, 319-345.

Shepard, R. N. (1972). Introduction to volume I. In R. Shepard, A. Romney, \& S. Nerlove (Eds.), Multidimensional Scaling: Theory and Applications in the Behavioral Sciences, Volume 1: Theory (pp. 1-20). New York: Seminar Press. Shepard, R. N. (1981). Psychophysical complementarity. In M. Kubovy \& J. Pomerantz (Eds.), Perceptual organization (pp. 279341). Hillsdale, $\mathrm{NJ}$ : Erlbaum.

Srivastava, R. K., Leone, R. P., \& Shocker, A. D. (1981). Market structure analysis: Hierarchical clustering of products based on substitution-in-use. Journal of Marketing, 45, 3848.

Sujan, M. (1985). Consumer knowledge: Effects on evaluation strategies mediating consumer judgment. Journal of Consumer Research, 12, 31-46.

Wold, H. (1982). Systems under indirect observation using PLS. In C. Fornell (Ed.), A second generation of multivariate analysis: Methods (pp. 325-347). New York: Prager. 